\title{
Resonant Photoemission Spectroscopy of Cu(InGa)Se 2 Materials for Solar Cells
}

\author{
V. I. Grebennikov ${ }^{a}$, T. V. Kuznetsova ${ }^{a}$, and M. V. Yakushev ${ }^{b}$ \\ ${ }^{a}$ Institute of Metal Physics, Ural Branch, Russian Academy of Sciences, Yekaterinburg, 620990 Russia \\ ${ }^{b}$ Ural Federal University, Yekaterinburg, 620002 Russia \\ e-mail: greben@imp.uran.ru
}

\begin{abstract}
The electron structure of $\mathrm{CuIn}_{1-x} \mathrm{Ga}_{x} \mathrm{Se}_{2}$ single crystals is determined via resonant photoemission and the main regularities of its transformation upon varying concentration $x$ from 0 to 1 are established. The dependence of the shape of valence band spectra on the photon energy is studied. Integral photoemission intensities are shown to be determined by atomic photoionization cross sections. Processes of the direct and two-step creation of photoelectrons accompanying photoemission and the participation of internal states in the spectra of electrons from valence bands are studied. Two-hole final states in photoemission are obtained upon threshold excitation of the $\mathrm{Cu} 2 p$ level. The strong interaction of holes leads to the multiplet splitting of these states. Partial densities of the components' states are determined using the energy dependence of atomic photoionization cross sections.
\end{abstract}

DOI: $10.3103 / \mathrm{S} 1062873813090153$

\section{INTRODUCTION}

The $\mathrm{CuIn}_{1-x} \mathrm{Ga}_{x} \mathrm{Se}_{2}$ (CIGS) semiconductors are used as the absorbing layer in solar cells. Thin-film devices based on them are leaders in the efficiency $(20 \%)$ of solar energy transformation, operation stability, and radiation resistance $[1,2]$. A great deal of attention is now being given to measuring the size of the energy gap using optical absorption or reflection spectra [3, 4]. Among theoretical calculations, those in which CIGS electron structure was studied according to zone were outstanding [5]. The problem is finding how this gap is formed and what has to be done to change it. How do we lower the recombination of electrons and holes generated by light? To answer this question, we must first know the distribution of electron states not only at the top of the valence band but throughout its depth.

The need to perform comprehensive studies of the electron structure of these compounds is beyond question, since it creates all of the necessary prerequisites for a detailed analysis of the formation of optical properties. The main factor constraining such investigations is the difficulty of preparing a perfect surface of samples; this is absolutely necessary since electrons emit only from a limited number of atomic layers. We can overcome this difficulty by using photoemission in the hard X-ray range $(3-8 \mathrm{keV})$, where the depth of electron output reaches ten unit cells [6]. Another possibility is to prepare films directly before measuring, which is how angle-resolved photoemission spectra of CuInSe ${ }_{2}$ were obtained in [7].

In this work, X-ray photoemission spectra (XPS) were obtained using synchrotron radiation with pho- ton energies that varied from 50 to $1200 \mathrm{eV}$. The energy resolution of the device was $0.1 \mathrm{eV}$. Our perfect working surface was prepared by cleaving single-crystal samples directly in the ultra-high vacuum chamber of a spectrometer.

\section{ORDER AND DISORDER OF INDIUM AND GALLIUM}

XP spectra (intensity $I$ of photoelectron output as a function of bond energy $E$ ) of the valence bands of the $\mathrm{CuIn}_{1-x} \mathrm{Ga}_{x} \mathrm{Se}_{2}$ system were obtained for different concentrations of gallium $x=0,0.05,0.1,0.25,0.5$, and 1 at two photon energies, 600 and $120 \mathrm{eV}$. A feature of these spectra is the presence of a narrow $\mathrm{Ga} 3 d$ band (bond energy, $19.5 \mathrm{eV}$ ) and double In $4 d$ band $(17.5$ and $18.5 \mathrm{eV})$; their intensities are directly determined by the oncentrations of the components. The valence bands of all the compounds are nearly identical; they resemble the $\mathrm{CuInSe}_{2}$ spectra presented in Fig. 1. The $\mathrm{CuGaSe}_{2}$ spectrum has a higher arm at bond energy $E=1 \mathrm{eV}$ and a more extended and powerful peak at $7.5 \mathrm{eV}$. The fine structure of compounds with $x=0.05$ and 0.1 is blurred, compared to compounds of 0.25 and 0.5 . This is a consequence of disordering in the alternation of $\mathrm{Ga}$ and In atoms at small concentrations and their ordering beginning with $x=0.25$.

\section{PHOTOIONIZATION CROSS SECTIONS}

We studied the dependence of XP spectra on photon energy $h v$. Figure 1 shows spectra $I(E)$ of the valence band of $\mathrm{CuInSe}{ }_{2}$ compound obtained at exci- 


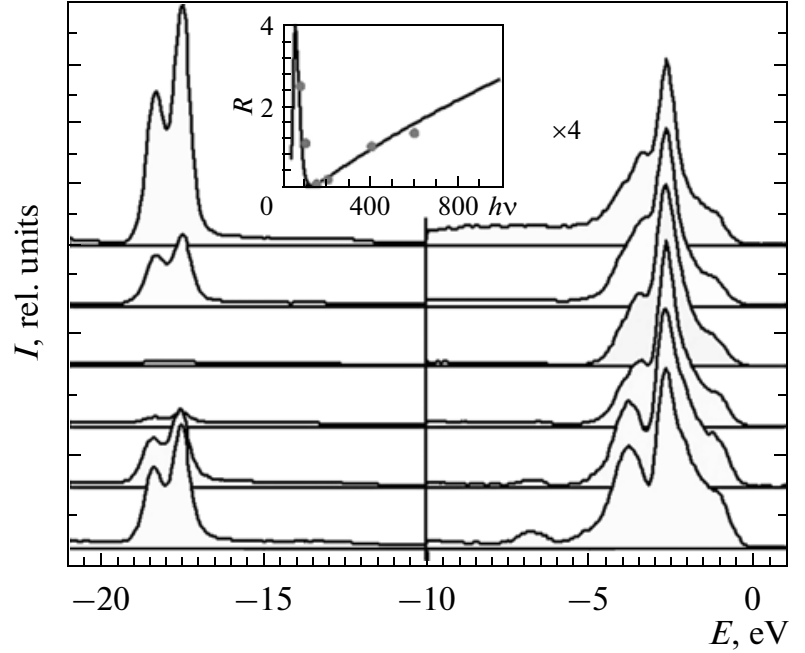

Fig. 1. XP spectra $I$ of the $\mathrm{CuInSe}{ }_{2}$ compound as functions of bond energy $E$ obtained at excitation energies $h v$ of (from top to bottom) 75, 100,150, 200, 400, and $600 \mathrm{eV}$. The insert presents the ratio of areas of the In $4 d$ band and valence band (dots) and the ratio $R$ of the calculated atomic photoionization cross sections In $4 d / \mathrm{Cu} 3 d$ (solid curve).

tation energies $h v$ of $75,100,150,200,400$, and $600 \mathrm{eV}$. The $4 d$ band of indium $(17.5$ and $18.5 \mathrm{eV})$ is close to these. It falls sharply in the region $h v \approx 150 \mathrm{eV}$. The ratio of the area of the $4 d$ band to the area of the valence band is shown by dots in the insert of Fig. 1 . Comparison to atomic calculations of photoionization cross sections of the In $4 d$ and $\mathrm{Cu} 3 d$ states (solid curve) shows that integral ionization cross sections in the crystal are described quite well by cross sections calculated for free atoms. Note the dependence of the shape of valence band spectra on the excitation energy. The spectrum obtained at $100 \mathrm{eV}$ has a single maximum; the XP spectrum taken at $600 \mathrm{eV}$ has two peaks. This difference is due to different energy dependences of the ionization cross sections for copper and selenium atoms. We use this below to determine the partial densities of states.

\section{RESONANCE: THE TWO-HOLE STATE}

Let us consider the behavior of the XP spectrum upon crossing the excitation threshold of the $2 p$ level of copper. X-ray absorption at the threshold increases by $20 \%$ (see the $L_{23}$ absorption spectrum of $\mathrm{Cu}$ in Fig. 2a). A photon transforms a $2 p$ electron into an unoccupied state with energy $E^{*}$. Additional scattering channels are generated by the $2 p$ hole that simultaneously appears. An excited electron can return to the initial $2 p$ state. The energy that is released is transferred to a valence electron that is undistinguishable from the valence electron that absorbed the photon. The total intensity in this case is determined from the square of the sum of the amplitudes of two transitions.

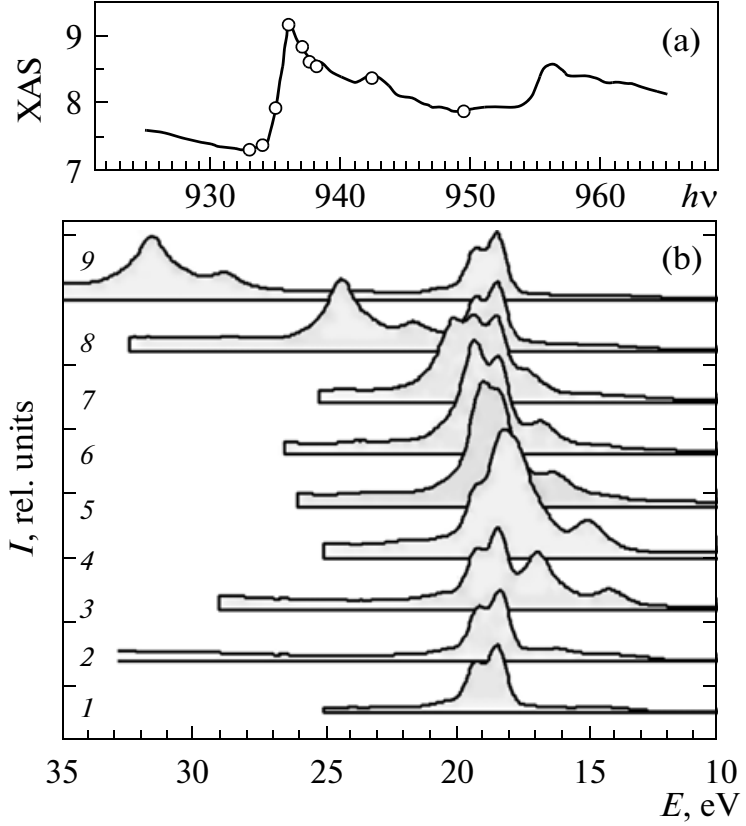

Fig. 2. (a) $\mathrm{Cu} L_{23}$ XAS absorption spectrum of our $\mathrm{CuIn}_{0.9} \mathrm{Ga}_{0.1} \mathrm{Se}_{2}$ compound and (b) XP spectra $I(E)$ at photon energy $h v$ equal to (from top to bottom) 933, 934, 935, 936, 937, 937.5, 938.2, 942.4, and $949.5 \mathrm{eV}$ (dots in Fig. 2a). The two-hole state (14 and $17 \mathrm{eV})$ is formed at a photon energy of $935 \mathrm{eV}$ (curve 3); its signal moves (in the scale of the bond energy) in proportion to the growth of $h v$.

This amplifies the XP spectrum of the valence band at the resonance frequency $[8,9]$. In the second channel, a $2 p$-hole is filled with a valence electron and the energy is transferred to a second valence electron that is detected as a photoelectron. The final state of the photoemission process contains two holes in the valence band, preferably on one copper atom (the wave functions in the Auger transition must overlap).

We write the energy conservation law in the final state:

$$
h v=E_{k i n}+E^{*}-E_{V}-E_{V},
$$

where $E_{V}$ is the valence state energy. If excitation occurs with conservation of energy $E^{*}=h v+E_{2 p}$ (which is not necessary for the nonstationary intermediate state), we obtain the following equality for the photoelectron kinetic energy:

$$
E_{k i n}=E_{V}+E_{V}-E_{2 p} \text {. }
$$

If the system has a long-lived localized excited state (a trap), the photoelectron energy is determined by the energies of a photon and an additional electron-hole pair:

$$
E_{k i n}=h v+E_{V}+E_{V}-E^{*} .
$$

To know which scenario is observed in our objects, let us consider the XP spectra. Note that the valence band spectra (in the interval of $0-10 \mathrm{eV}$ ) respond little to a change in photon energy; for this reason, only the part of bond energies of $10-35 \mathrm{eV}$ is shown in Fig. 2. 
In curve $1(h v=333 \mathrm{eV})$, a single indium line is seen. A new line consisting of two peaks of 14 and $17 \mathrm{eV}$ is created in curve 2 and formed in curve $3(h v=335 \mathrm{eV})$. This line moves in the scale of bond energies $\left(-E_{B}=E_{k i n}-h v+U\right.$, where $U$ is the work function) and the constant kinetic energy determined by equality (2). When the Auger transition and In $4 d$ bands are combined (curves 4 and 5), the spectrum intensity increases by a factor of 2 .

There are thus no long-lived excited states (electron traps) on copper atoms in CIGS; the energy is conserved at all stages of the photoemission process, and we observe the creation of an Auger line at the $\mathrm{Cu} L_{3}$ threshold in Fig. 2b. A strongly correlated twohole state is formed that has a clear multiplet structure consisting of two main peaks. Two more final photoemission states can be seen: a photohole on any atom in the valence band (Fig. 1, the curve at $h v=600 \mathrm{eV}$ ) and one photohole on a copper atom in the band due to the large ionization cross section (Fig. 1, the curve at $h v=$ $100 \mathrm{eV})$.

\section{PARTIAL DENSITIES OF STATES}

In [10], the state density was separated into two contributions from the $\mathrm{Cu}$ and $\mathrm{Se}$ atoms. Let us examine the problem of determining the partial densities of states from the XP spectra. The rate of detecting photoelectrons $I_{i}(e)$ with bond energy $e$ upon energy $h v_{i}$ excited by photons is determined from the sum of the products of partial photoionization cross sections $b_{i j}$ and densities of states $d_{j}(e)$ of the corresponding symmetry (index $j$ ):

$$
k_{i} \sum_{j} b_{i j} d_{j}(e)=I_{i}(e) .
$$

Coefficients $k_{i}$ are proportional to the flux of photons with a particular energy. Cross sections $b_{i j}$ are known from atomic calculations, while spectra $I_{i}(e)$ are taken from the experiment. It is required to find unknown partial densities of states $d_{j}(e)$. Since the quantity of electrons included in atomic cross sections, the integral partial density of states is equal to unity: $\int d_{j}(e) d e=1$. It should be remembered that equalities (4) are approximate; therefore, the system can be overdetermined (the number of unknowns is greater than that of equations). The solution is obtained by minimizing the sum of the squared differences between the leftand right-hand sides of equalities (4). Integration with respect to the photoelectron energy yields the atomic limit

$$
k_{i} \sum_{j} b_{i j}=S_{i}=\int \operatorname{deI}_{i}(e)
$$

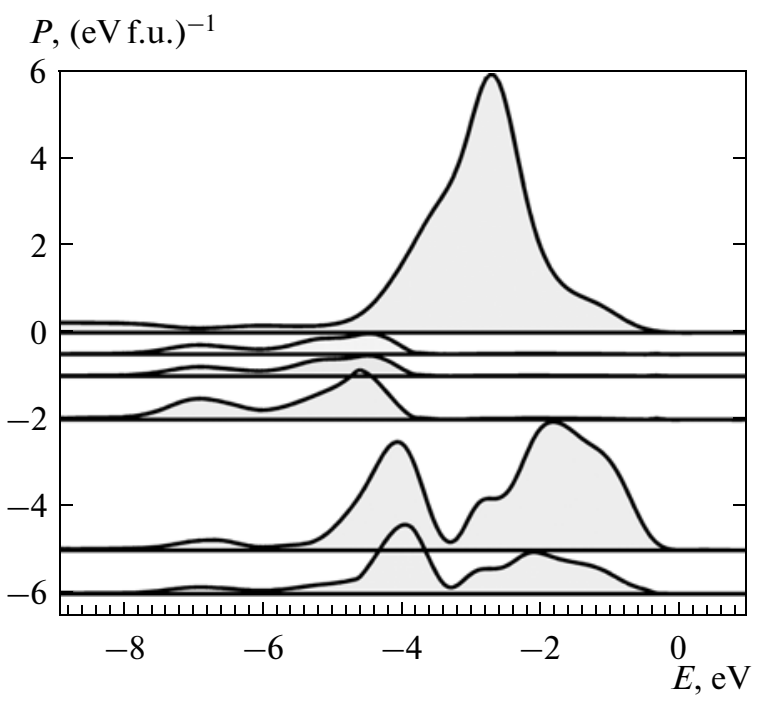

Fig. 3. Partial densities of states $P$ of $\mathrm{CuInSe}_{2}$ valence bands (from top to bottom): $d \mathrm{Cu}, s \mathrm{Cu}, p \operatorname{In}, s \mathrm{In}, p \mathrm{Se}$, and $s \mathrm{Se}$, obtained from the experimental spectra (Fig. 1).

Dividing equality (4) by (5), we eliminate the unknown coefficients $k_{i}$ and obtain the equations

$$
\sum_{j} c_{i j} d_{j}(e)=f_{i}(e)
$$

where

$$
c_{i j}=\frac{b_{i j}}{\sum_{j} b_{i j}} \text { and } f_{i}(e)=\frac{I_{i}(e)}{S_{i}} .
$$

The positive coefficients $c_{i j}$ determine ourprobabilities. The weighted mean value of the partial density of states is equal to the normalized XP spectrum obtained at a given photon energy. Solving problem (6) for a set of positive functions, we find the unknown partial state densities: $P=n_{j} d_{j}(e)$.

Figure 3 shows partial state densities $P(E)$ of $\mathrm{CuInSe}{ }_{2}$ compound, calculated from the experimental spectra of the valence band (Fig. 2). The ten $d$-states of copper lie in the interval of $0-4.5 \mathrm{eV}$ with the maximum energy of $2.7 \mathrm{eV}$. They displace other states from its vicinity. The eight $p$ - and four $s$-states of selenium are positioned higher and lower than the copper states. Three $s-p$-states of indium are concentrated in the lower part of the valence band.

\section{SUPERSTOICHIOMETRIC INDIUM}

Compounds that deviate from the ideal $\mathrm{CuInSe}_{2}$ formula are of great practical importance. Adding several percent of indium considerably improves the mechanical properties of the material. Our study revealed an interesting feature of photoemission from compounds with superstoichiometric (5 and 10\%) indium: low-energy (up to $300 \mathrm{eV}$ ) photoelectrons undergo extremely strong inelastic scattering, while 
electrons of higher energies emit from the substance without visible losses. We may assume that low-energy electrons are scattered when passing through the film created on a sample's surface by excess metal.

\section{ACKNOWLEDGMENTS}

Our experiments were conducted at the RussianGerman laboratory of the BESSY II synchrotron with the technical support of O. Vilkov. This work was supported by the Russian Foundation for Basic Research, projects nos. 11-02-00379, 11-02-0093, and 11-0201221; and by the Ural Branch of the Russian Academy of Sciences, project no. 12-U-2-1002.

\section{REFERENCES}

1. Chopra, K.L., Paulson, P.D., and Dutta, V., Prog. Photovolt: Res. Appl., 2004, vol. 12, p. 69.

2. Bagnall, D.M. and Boreland, M., Energy Policy, 2008, vol. 36, p. 4390.
3. Sung-Ho Han, Persson, C., Hasoon Falah, S., et al., Phys. Rev. B, 2006, vol. 74, p. 085212.

4. Rincon, C., Wasim, S.M., Marin, G., and Molina, I., J. Appl. Phys., 2003, vol. 93, p. 780.

5. Zhang, S.B., Su-Huai Wei, Zunger, A., and KatayamaYosida, H., Phys. Rev. B, 1998, vol. 57, p. 9642.

6. Gloskovskii, A., Jenkins, C.A., Ouardi, S., et al., Appl. Phys. Lett., 2012, vol. 100, p. 092108.

7. Hofmann, A. and Pettenkofer, C., Phys. Rev. B, 2011, vol. 84, p. 115109.

8. Yablonskikh, M.V., Yarmoshenko, Yu.M., Grebennikov, V.I., et al., Phys. Rev. B, 2001, vol. 63, no. 23, p. 235117.

9. Grebennikov, V.I., Poverkhn. Rentgen., Sinkhrotron. Neitron. Issl., 2002, no. 11, p. 41.

10. Löher, T., Klein, A., Pettenkofer, C., and Jaegermann, W., J. Appl. Phys., 1997, vol. 81, p. 7806.

Translated by A. Nikol'skii 